fuged and the supernatant discarded; the packed mass was suspended in the undiluted immune serum and left to stand for $1 \mathrm{hr}$. at $37^{\circ} \mathrm{C}$, and for $2 \mathrm{hr}$. at $4^{\circ} \mathrm{C}$. ; after this the gel-antigen complex was centrifuged off, removing the heterologous antibody.

The centrifuged sediment of about $5 \mathrm{ml}$. antigen-gel suspension was sufficient to absorb one heterologous antibody from $50 \mathrm{ml}$. undiluted rabbit serum. If the immune serum contains more than one heterologous antibody, they can be absorbed in one operation by previously mixing the necessary antigen suspensions.

The titre of the homologous antibody remained unchanged after purification. We obtained specific monovalent precipitating sera by this method from all of 51 rabbits which were immunized against human, horse, cow, p:g and dog serum proteins. JosEPH BöszöRMÉNYI

'Human' State Vaccine Institute

for Production and Research,

X. Szállás-u 5-7, Budapest.

2 Proom, H., J. Path. Bact., 55, 419 (1943).

"Kabat-Mayer, "Experimental Immunochemistry" (C. C. Thomas, Springfield, 1948).

Haurowitz, F. S., Tekman, S., Bilen, M., and Schwerin, P., Biochem. J., 41, 305 (1949)

- Campbell, D. H. Luescher, E., and Lerman, L. S., Proc. U.S. Nat. Acad. Sci., 87, 575 (1951)

- Isliker, H. C., Ann. N.Y. Acad. Sci., 57, 225 (1953).

\section{Bacterial Dissimilation of Indoleacetic Acid : a New Route of Breakdown of the Indole Nucleus}

AN organism capable of breaking down indoleacetic acid has been isolated in pure culture and characterized as a member of the genus Pseudomonas (Migula), but it does not correspond to any of the species described in the sixth edition of "Bergey's Manual of a Determinative Bacteriology".

Percolation of $10 \mathrm{gm}$. of soil resulted in logarithmic decomposition of $10^{-3} M$ indoleacetic acid after an initial lag of approximately seven days; further additions of $10^{-3} M$ indoleacetic acid were decomposed without a lag at a maximal rate of $0.24 \mathrm{mgm}$./ hr./gm. of soil.

With standard manometric techniques the adaptation to indoleacetic acid was shown to involve simultaneous adaptation to skatole (3-methyl-indole), indoxyl (3-hydroxy-indole), salicylic acid and catechol. No adaptation had occurred to indole, anthranilic acid, o-amino-phenol, o-nitrophenol, o-nitrobenzoic acid or phthalic acid. The initial step in the dissimilation appears to be a decarboxylation, evolution of carbon dioxide slightly preceding the uptake of oxygen.

For aerated cultures, in nitrogen-free medium supplemented with $10^{-2} M$ indoleacetic acid, skatole, indoxyl compounds, salicylic acid and catechol were identified by chromatography on paper. Catechol was isolated as a crystalline compound (melting point, $103^{\circ} \mathrm{C}$.) from an ethereal extract of the acidified culture fluid. The sequence of appearance of intermediates was consistent with a route via $(a)$ an initial decarboxylation of indoleacetic acid to yield 3-methyl-indole, (b) replacement of the methyl group by an hydroxyl group, $(c)$ hydroxylation and reduction to give a 2,3-dihydroxy-dihydro-indole, (d) splitting of the ring followed by $(e)$ hydrolysis to liberate ammonia and the formation of salicylic acid, which is then metabolized through catechol. The ammonia was converted to nitrite and nitrate.
This scheme shows features common to previously postulated routes for aromatic breakdown ${ }^{1,2}$, but differs from those for tryptophan ${ }^{3}$ and indole, in that ring fission does not involve kynurenine or anthranilic acid formation.

I wish to thank Prof. F. S. Stewart for supervising this work, a full account of which will be published elsewhere.

Department of Bacteriology,

Michael H. Proctor*

Trinity College, Dublin. March 21.

* Present address: Department of Bacteriology, University of Wisconsin, Madison 6, Wisconsin.

${ }^{t}$ Rogoff, M. H., and Wender, I., J. Bact., 73, 264 (1957).

¿ Walker, N., and Wiltshire, G. H., J. Gen. Microbiol., 8, 273 (1953). ${ }^{3}$ Stanier, R. Y., and Tsuchida, M., J. Bact., 58, 45 (1949).

- Sakamoto, Y., Uchida, M., and Ichihara, K., Med. J. Osaka Oniv., 3, 477 (1953).

\section{Carbohydrate Peptide Complex from Egg Albumin}

ONE of us described in 1938 the preparation from crystalline egg albumin of a polysaccharide containing mannose and glucosamine and a small amount of an unidentified nitrogenous substance ${ }^{1}$. On the basis of hexose and amino-sugar estimations on the intact protein and the molecular weight of 40,000 then accepted for hen's egg albumin, it was concluded that one albumin molecule contains four mannose and two glucosamine residues. Since the method of isolation involved acetylation, no information could then be obtained as to whether the glucosamine residues were originally present in the N-rcetylated form. Since there were no suitable chromatographic methods available in 1938 , the work was discontinued and many problems concerning this carbohydrate were left unanswered, particularly the nature of the linkage of the polysaccharide with the peptide chain.

The problem has been taken up again in the last few years in this laboratory and some observations have been made which are here recorded. In the first place, the molecular weight of egg albumin now accepted ${ }^{2}$ is approximately 45,000 and the anslytical figures with respect to the carbohydrate composition have to be revised. Mannose estimation suggests that the hexose content is about 2 per cent, but the errors of the various methods used may be quite considerable. Similarly, the most reliable value for amino-sugar is 1.2 per cent (in terms of the hexosamine base). These figures indicate that egg albumin probably contains five mannose and three glucosamine units. We have also determined the volatile acids arising on hydrolysis of the protein with $2 \mathrm{~N}$ hydrochloric acid for various periods of time, and it could be shown by partition chromatography ${ }^{3}$ that approximately four molecules of acetic acid were produced. The conclusion that this acid arises largely from $\mathrm{N}$-acetyl-glucosamine residues was supported by the finding that hydrolysis of the complex peptide to be described below yields the acetylamino-sugar, which was identified by paper chromatography.

A peptide-carbohydrate complex was obtained by the following procedure. The heat-denatured protein was hydrolysed, first with pepsin at $p \mathbf{H ~} 2 \cdot 8$, then by a mixture of trypsin and chymotrypsin at $p \mathbf{I I} 7 \cdot 8$ and finally by a mould protease described by Crewther and Lennox ${ }^{4}$. The hydrolysate was chromatographed on a carbon-'Celite' column and the carbohydratecontaining material was obtained by gradient elution with ethanol-water. Final purification was carried 